\title{
Türkiye'de Para Talebi Fonksiyonu ve Faiz Oranının Simetrik Etkisi
}

\section{Mortaza OJAGHLOU ${ }^{1}$}

Öz

Merkez bankalarının belirledikleri hedeflere ulaşabilmeleri için etkin bir para politikasına sahip olmaları gerekmektedir. Etkin bir para politikasına sahip olmak için de istikrarlı ve öngörülebilir bir para talep fonksiyonu gerekmektedir. $\mathrm{Bu}$ bağlamda, bu çalı̧̧manın amacı doğrultusunda, 2005Q04-2019Q03 üç aylık verilerini kullanarak Türkiye'de faiz oranının para talebi fonksiyonuna olan asimetrik etkisi araştııılmıştır. $\mathrm{Bu}$ çalışmada (Greenwood-Nimmo, Shin, and Treeck 2010) ve (Shin, Yu, and Greenwood-Nimmo 2013) tarafindan geliştirilen Çoğaltıcı Doğrusal Olmayan ve NLARDL sinır testi (Nonlinear Autoregressive Distributed Lag Bound Test) yöntemi kullanılmıştır. Sonuçlara göre uzun dönemdeki gelir, faiz ${ }^{\text {pos }}$ oranı doğrusal ve pozitif yönde etkiler bırakırken faiz ${ }^{\text {neg }}$ oranı ve döviz kuru negatif yönde etkilemiştir. Ayrıca, tahmin edilen model bulguları etkin bir para politikası için istikrarlı para talebi fonksiyonunun gerekliliğini savunan monetarist yaklaşımı desteklemektedir ve Türkiye'de para talebinin istikrarlı olduğunu onaylamaktadır. İstikrarlı bir para talebi, merkez bankasının para politikasını kontrol ederek reel ekonomiyi etkileyebileceğini göstermektedir.

Anahtar Kelimeler: Para Talebi Fonksiyonu, Doğrusal Olmayan Otoregresif Dağıtılmış Gecikme Yaklaşımı (NARDL), Faiz Oranı

\footnotetext{
${ }^{1}$ Dr. Öğr. Üyesi. İktisadi ve İdari Bilimler Fakültesi, Ekonomi ve Finans bölümü, İstanbul Aydın Üniversitesi, mortazaojaghlou@aydin.edu.tr

Doi Num: 10.17932/IAU.FCPE.2015.010/fcpe_v06i1006
} 


\title{
Asymmetrical Effects Of Interest Rates On The Money Demand Function In Turkey
}

\begin{abstract}
Central banks need to have an effective monetary policy in order to achieve their goals. For having an effective monetary policy, a stable and predictable money demand function is required. In this context, purpose of this study is determined stability of money demand function and asymmetric effect of interest rates on the money demand function in Turkey over the period 2005Q04-2019Q03. For this goal we cried out non-linear autoregressive distributed lag approach (NARDL) developed by (Greenwood-Nimmo, Shin, and Treeck 2010) and (Shin, Yu, and Greenwood-Nimmo 2013). According to the results, the effect of interest rate ${ }^{\text {pos }}$ and income on long run money supply are positive. While interest rate ${ }^{\text {neg }}$ and exchange rate effect negatively. In addition, according the results money demand function in Turkey is stable and our finding and estimated function is consistent with monetarist approach which believes for haveing an effective monetary policy, a stable money demand function is required.
\end{abstract}

Keywords: Money Demand Function, Non-Linear Autoregressive Distributed Lag Approach (NARDL), Internet Rate

\section{GİRIŞ}

Faiz oranında meydana gelen değişiklikler piyasa temelli ekonominin önemli bir özelliğidir. Ulusal ve uluslararası meydana gelen herhangi bir ekonomik değişiklik faiz oranının değişimine sebebiyet verecektir (Gambacorta 2008a), (Chong, Liu, and Shrestha 2006). Bu bağlamda para talebi fonksiyonu tahmini, makroekonomik literatürde büyük önem taşımaktadır. Para politikasının başarısı para talebi fonksiyonu özelikleri ve istikrarına bağlıdır ve para talebi fonksiyonu para politikasının reel ve finans sektörü üzerinde nasıl etki bıraktığını açıklar (Poole 1970). Friedman (1966) çalışmasında faiz oranının, para talebi fonksiyonu ve para politikası üzerindeki önem ve etkisini incelemiştir. Parasalcı (Chicago) okulu para yaklaşımlarından sonra, para politikası ekonomide en önemli konulardan biri haline gelmiştir. Bu okula göre para politikası, gelir, enflasyon ve istihdam gibi reel ekonomi değişkenlerini etkileyen en etkili 
araçtır. Chicago okuluna göre etkin ve güçlü para politikası için istikrarlı ve öngörülebilir para talebinin mevcut olması gerekmektedir (Driscoll and Ford 1980).

Teorik çerçevede, bazı faktörler faiz oranının ayarlanmasında önemli rol oynamaktadır. Bu faktörler para politikası yönelimi, serbest veya kontrollü ekonomik rejimi, finansal yapı, kalkınma düzeyi, bankacılık sektörü yoğunlaşması, banka büyüklüğü, finansal piyasanın açıklık derecesi, asimetrik bilgi ve diğer faktörlerin yanı sıra menü maliyetleri vb. olarak sayılabilir (Gambacorta 2008b).

Para talebi teorisi genellikle Klasik para talebi ve Modern para talebi teorileri üzerine kuruludur (Stern 2006).

\section{KLASIK PARA TALEBİ TEORILERİ}

Klasik Miktar Teorisi; Klasik Miktar teorisi para talebi 19. ve 20. yüzyılın başlarında klasik iktisatçılar tarafından geliştirildi. Paranın miktar teorisi, toplam gelirin nominal değerinin nasıl belirlendiğine dair bir teoridir. Ancak klasik miktar teorisinin en önemli özelliği faiz oranlarının para talebi üzerinde bir etkisi olmamasıdır (Mishkin 2004).

Miktar teorisi M.V = P.T; olarak tanımlanmıştır. M nominal para arzı, V; işlem hızı ve P; fiyat seviyesi, T; işlem seviyesidir. Fisher (1911) kısa vadede $\mathrm{T}$ ve V'nin sabit olmasını destekler. Böylece para arzındaki bir artış, fiyat seviyesini de arttıracaktır. Döviz denklemi, işlemlerin seviyesi ile orantilidir.

Cambridge Miktar Teorisi: miktarı teorisinin Cambridge yaklaşımı, Cambridge'deki Marshal (1920) ve Pigou (1917) gibi ekonomistler tarafından geliştirilmiştir. Laidler (1993)'a göre Fisher'ın miktar teorisi ile Cambridge yaklaşımı arasındaki temel farklar, para talebinin farklı belirleyicileri olan bireysel davranışlardır. Cambridge yaklaşımında, insanlar için para tutmanın, belirli faktörlere, düşüncelere, kişisel servete, diğer varlıkların getirisine veya geleceğe ilişkin beklentilerine bağlı olarak bireyin para tutmayla elde ettiği faydaya bağlıdır. 
Keynesyen Para Talebi Teorisi: Keynes (1936)'e göre Keynesyen para politikası ve aktarım mekanizması, faiz üzerinden dolaylı işlemektedir. Bu yüzden Keynesyen aktarım mekanizması dolaylı aktarım mekanizması olarak literatürde yer almaktadır. $\mathrm{Bu}$ yaklaşımda para politikası faiz oranlarına tesir ederek yatırım oranını etkilemektedir. Para talebi fonksiyonu likidite tuzağı yani faiz esnekliği sonsuz olduğu ve yatırımların faiz esnekliği sıfır olduğu durumda Keynesyen aktarım mekanizması işlevsiz hale dönüşecektir. Bu durumda faiz politikası reel ekonomiye etki etmeyecek ve reel etkiler doğurmayacaktır.

\section{MODERN MIKTAR TEORÍSI}

Milton Friedman (1956), "Paranın Miktar Teorisi: Yeniden Düzenleme" makalesinde klasik miktar teorisini tekrardan ele alarak modern para talebi teorisi geliştirmiştir. Friedman teorisinde Irving Fisher ve miktar teorisini ele almıştır. Ancak Friedman'ın para talebi analizi daha çok Keynes'in analizine benzemektedir. (Mishkin 2004).

Friedman para talebi şu şekilde yazılabilir:

$M_{d} / P=f\left(Y_{p}, r_{b}-r_{m}, r_{e}-r_{m}, \pi_{e}-r_{m}\right)$

$\mathrm{M}_{\mathrm{d}} / \mathrm{P}$ : reel para dengesi talebi,

$\mathrm{Y}_{\mathrm{P}}$ : sürekli gelir,

$\mathrm{r}_{\mathrm{m}}$ : Beklenen para getirisi,

$\mathrm{r}_{\mathrm{b}}$ : Beklenen tahvil getirisi,

$\mathrm{r}_{\mathrm{e}}$ : Beklenen öz kaynak getirisi

$\pi_{\mathrm{e}}$ : Beklenen enflasyon oran 1

\section{LITERATÜR TARAMASI}

Son yıllarda gerek ulusal gerekse uluslararası literatür incelendiğinde para talep fonksiyon tahminine yönelik çalışmalar daha fazla ilgi görmüştür. $\mathrm{Bu}$ çalışmalar genellikle VECM ve eşbütünleşik analizi ile analiz edilmiştir. Tablo 1'de gelişmekte olan ülkelerde para talebi ile ilgili literatürün kısa bir incelemesi bulunmaktadır; 
Tablo 1: Literatür Özeti

\begin{tabular}{ccc}
\hline Araştırmacı (lar) & Ülke & Metod \\
\hline (Akinkunmi 2004) & Gelişmekte Olan Ülkeler & ARDL \\
(Komijani ve Nazarian 2004) & İran & Cointegration ve ECM \\
(Shahrestani ve Sharifi-Renani & İran & ARDL \\
2007) & Nijerya & ARDL \\
(Akinlo 2006) & Gelişmekte Olan Ülkeler & ARDL \\
(Tanku 2005) & (20 Adet) & ARDL \\
Bazi Asya Ülkeleri & \\
(Bahmani-Oskooee ve Rehman & Sundi Arabistan & ARDL \& Granger- \\
(Al-Badi 2002) & & Causality \\
(Alsahafi 2009) & Suudi Arabistan & Cointegration ve ECM \\
(Liao 2005) & Kanada & Cointegration ve ECM \\
(Bahmani-Oskooee 1996) & Iran & ECM \\
(Dritsakis 2012) & Yunanistan & Cointegration (JJ \\
(Halicioglu and Ugur 2005) & Türkiye & TEST) \\
(Akinkunmi 2004) & Gelişmekte Olan Ülkeler & ARDL \\
\hline & & ARDL \\
\hline
\end{tabular}

\section{MODEL, VERI SETİ VE YÖNTEM}

Türkiye için para talebinin belirlenmesi ve faiz oranının bu fonksiyon üzerinde asimetrik etkisini analiz etmek üzere daha önce (BahmaniOskooee 2001), (Akinlo 2006) ve (Dritsakis 2012) tarafından kullanılmış olup model çalışmada aşağıdaki gibi ele alınmıştır:

$$
\mathrm{Lm}_{2} / \mathrm{p}=(\text { GSYIH, faiz, Lkur })
$$

Çalışma amacına ilişkin 1. modelle ilgili NARDL modeli:

$$
\mathrm{Lm}_{2} / \mathrm{p}=\left(\text { faiz }^{\mathrm{POS}}, \text { faiz }^{\mathrm{NEG}}, \mathrm{LGSYİH}, \mathrm{LEx}\right)
$$


$\mathrm{Lm}_{2} ; \mathrm{m}_{2}$ Para Arzının Logaritması,

P: enflasyon oranı

$\mathrm{Lm}_{2} / \mathrm{p}$ : Reel para arz1

Faiz: Kısa Dönem Faiz Oranı,

LGSYİH: GSYİH'nin logaritmas1,

LEx: Dolar/TL Nominal Döviz Kuru Logaritması,

Türkiye para talebi tahmin fonksiyonu için ele alınan model 1'de kullanılan seriler 2005Q4- 2019Q4 yıllarına ilişkin çeyrek veriler Türkiye Cumhuriyet Merkez Bankası'nın elektronik veri dağıtım sistemin (EVDS)'den alınmıştır.

\section{Birim Kök Testi}

Literatürde çeşitli durağanlık testlerin arasında en çok ve yaygın olan test Genişletilmiş Dickey-Fuller (Augmented Dickey Fuller: ADF) ve PhillipsPron: PP testidir.

Tablo 2: Birim Kök Testi Sonuçları

\begin{tabular}{ccccc}
\hline Değişken & \multicolumn{2}{c}{ ADF } & \multicolumn{2}{c}{ PP } \\
\hline & Sabitli & Sabitli ve Trendli & Sabitli & Sabitli ve Trendli \\
\hline P & $-1.57^{*}$ & -1.83 & $-3.75^{* * *}$ & $-3.43 * *$ \\
GSYİH & -0.56 & -2.01 & -0.59 & -2.01 \\
$\Delta$ GSYİH & $-6.4 * * *$ & $-6.42 * * *$ & $-6.50 * * *$ & $-6.44 * * *$ \\
M2 & 5.28 & 1.61 & 10.00 & 2.64 \\
$\Delta$ M2 & $-3.41 * *$ & $-10.46^{* * *}$ & $-7.92 * * *$ & $-10.47 * * *$ \\
Faiz & $-5.3 * * *$ & $-3.32^{*}$ & $-4.83 * * *$ & $-3.16^{*}$ \\
EX & 5.452 & 3.123 & 6.98 & 2.61 \\
$\Delta \mathbf{E X}$ & $-6.08^{* *}$ & $-5.39 * * *$ & $-6.09 * * *$ & $-6.90^{* * *}$ \\
\hline
\end{tabular}

$* * *, * *$ ve * sırasıyla $\% 99, \% 95$ ve $\% 90$ güvenlik aralığını göstermektedir. 
$\mathrm{Bu}$ testler sonucunda Tablo 2'de görüldüğ̈̈ üzere serilerin tamamının I(0), I(1) seviyesinde durağan olması sebebiyle NARDL asimetrik testi yapılması yönünde hiç bir engel teşkil etmemektedir. NARDL yöntemi serilerin I (0) ve I (1) kombinasyonlarının aynı denklem içerisinde kullanılmasına izin verir. Bu nedenle, faiz oranının para arzı üzerinde asimetrik etkisini para talep fonksiyonu çerçevesinde NARDL modelini kullanarak analiz edeceğiz.

[(Greenwood-Nimmo, Shin, and Treeck 2010)] ve (Shin, Yu, and Greenwood-Nimmo 2011) tarafından geliştirilen ve simetrik uzun ve kısa dönemli doğrusal olmayan ilişkiyi ve asimetrik hata düzeltmeyi birleştiren NARDL modeli ile yapılandırılmış kısmi toplam ayrışma aşağıdaki gibi sunulmaktadır:

$y_{t}=\beta^{+} x_{t}^{+}+\beta^{-} x_{t}^{-}+u_{t}$

$\mathrm{xt}: \mathrm{k} \times 1$ temsil eden vektör ve $x_{t}=x_{0}+x_{t}^{+}+x_{t}^{-}$

ve terimler $\mathrm{x}$ 'deki pozitif terimler $\mathrm{x}$ 'deki pozitif ve negatif serilere ayrılmış değişkenlerdir;

$x_{t}^{+}=\sum_{j=1}^{t} \Delta x_{j}^{+}=\sum_{j=1}^{t} \max \left(\Delta x_{j}, 0\right), x_{t}^{-}=\sum_{j=1}^{t} \Delta x_{j}^{-}=\sum_{j=1}^{t} \min \left(\Delta x_{j}, 0\right)$

Modelle ilgili hata düzeltme terimi ise:

$\Delta y_{t}=\rho y_{t-1}+\theta^{+} x_{t-1}^{+}+\theta^{-} x_{t-1}^{-}+\sum_{j=1}^{p-1} \gamma_{j} \Delta y_{t-j}+\sum_{j=0}^{q}\left(\pi_{j}^{+} \Delta x_{t-j}^{+}+\pi_{j}^{-} \Delta x_{t-j}^{-}\right)+\varepsilon_{t}$,

Ve Sifir hipotez: $\rho=\theta$ pos $=\theta$ neg $=0$

Sistemin uzun dönem asimetrik ve kümülatif dinamik çarpanlarına bağlı steady state ise

$$
\begin{aligned}
& m_{h}^{+}=\sum_{j=0}^{h} \partial y_{t+j} / \vartheta x_{t}^{+} \\
& m_{h}^{-}=\sum_{j=0}^{h} \partial y_{t+j} / \vartheta x_{t}^{-} \quad \mathrm{h}=0,1,2, \ldots
\end{aligned}
$$


Model K da ilgili NARDL modeli ise

Bizim çalışmamızla ilgili olarak Asimetrik ARDL (NLARDL) aşağıdaki gibi yazılmaktadır:

$$
\begin{aligned}
& \Delta I M_{d t}= \\
& =\alpha_{0}+\sum_{q=1}^{p 1} \alpha_{1 q} \Delta l M_{S i, t-q}+\sum_{q=0}^{p 2} \alpha_{2 q} \Delta i_{i, t-q}^{p o s}+\sum_{i, t-1}^{p 3} \alpha_{q=0} \Delta i_{i, t-q}{ }_{i, t-1}^{n e g}+\sum_{q=0}^{p 4} \alpha_{4 q} \Delta G D P_{i, t-q}+\sum_{q=0}^{p 5} \alpha_{5 q} \Delta E X_{i, t-q}+ \\
& \sum_{q=0}^{p 5} \alpha_{6 q} \Delta P_{i, t-q}++\beta_{1} I M_{S i, t-1}+\beta_{2} G D P_{i, t-1}+\beta_{3} E X_{i, t-1}+\beta_{4} P_{i, t-1}+\beta_{5} i^{p o s}{ }_{i, t-1}+\beta_{6} i^{n e g}{ }_{i, t-1}+\varepsilon_{t}
\end{aligned}
$$

\begin{tabular}{|c|c|c|}
\hline \multirow[t]{2}{*}{ Değisşkenler } & \multicolumn{2}{|c|}{ NLARDL Model $(3,0,0,0,0)$} \\
\hline & $\begin{array}{l}\text { Uzun Dönem } \\
\text { Katsayıları }\end{array}$ & $\begin{array}{l}\text { Değişsenler ile } M_{d} \text { arasında kısa } \\
\text { dönem iliş̧i (f-stat) }\end{array}$ \\
\hline C & $14.11 * * *$ & -- \\
\hline$i^{p o s}$ & 0.022 & $\begin{array}{l}4.07^{* *} \text { (kısa dönem ilişki } \\
\text { mevcuttur) }\end{array}$ \\
\hline$i^{n e g}$ & $-0.059 * * *$ & 0.75 (kısa dönem ilişki yoktur) \\
\hline LGSYİH & $2.6 * 10-11 *$ & 11.58 (kısa dönem ilişki mevcuttur) \\
\hline LEX & $-0.26^{* *}$ & $\begin{array}{l}23.04 * * * \text { (kısa dönem ilişki } \\
\text { mevcuttur) }\end{array}$ \\
\hline F-Bounds & $5.52 * * *$ & -- \\
\hline $\mathbf{E C}_{\mathrm{t}-1}$ & $-0.83 * * *$ & -- \\
\hline $\begin{array}{l}\text { Breusch- godfrey- } \\
\text { Serial Correlation LM }\end{array}$ & $\mathrm{F}=1.44(0.24)$ & -- \\
\hline
\end{tabular}

Söz konusu denklemin tahmin sonuçları aşağıdaki tabloda özetlenmiştir.

Tablo 3: Para Talebine Direkt Kısa ve Uzun Dönem Etkisi

$* * *, * *$ ve $*$ sirasılyla $\% 1, \% 5$ ve $\% 10$ anlamlılık düzeylerini ifade etmektedir.

Tablo 3'te özetlenen uzun ve kısa dönemli NARDL tahminleri bilgileri 1şığında reel para talebi ile reel gelir, faiz ve döviz kuru değişkenleri arasında uzun ve kısa dönemli eşbütünleşme ilişkisinin mevcut olduğunu 
göstermektedir. Elde edilen bulgulara göre $\left(i^{\text {pos }}\right.$ katsayısı hariç) katsayılar istatistiksel olarak anlamlıdırlar. Katsayı işaretleri ve etkileşim yönleri açısından döviz kuru katsayısı teorik beklentilerle uyum sağlamaz iken diğer katsayılarla uyum sağlamaktadır. Ancak faiz oranının çarpan (multiplier) NARDL etkisine baktığımızda $i^{\text {pos }}$ reel para arzı üzerinde daha çok negatif iken $i^{\text {neg }}$ etkisi daha çok pozitif olmuştur ancak $i^{\text {pos }} i^{\text {neg }}$ etkisi genelde pozitif olduğunu görmekteyiz.

Grafik 1: Faiz Oranının Para Talebi Fonksiyon Üzerinde Simetrik ve Multiplier Etkisi

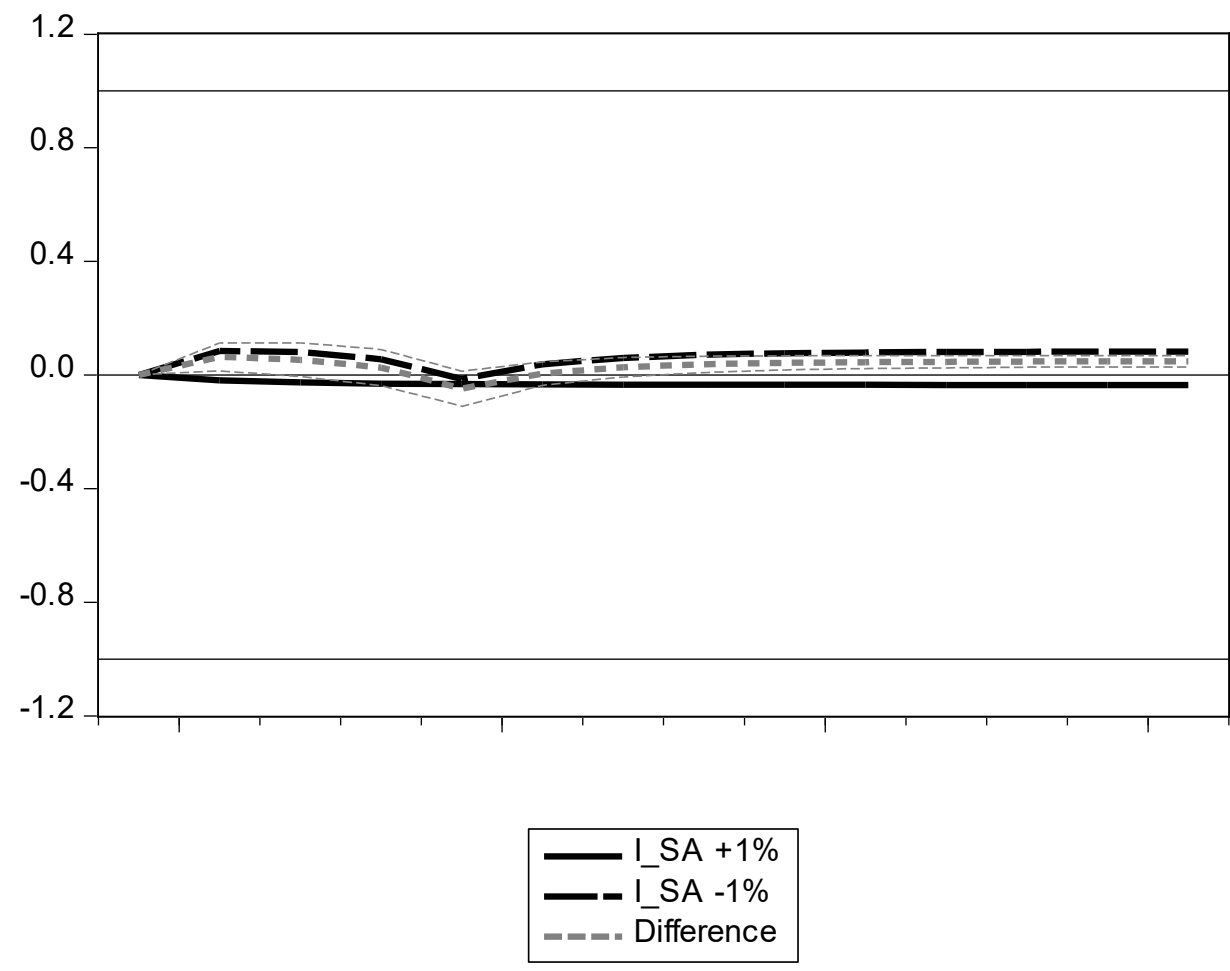

Breusch- Godfrey- Serial Correlation LM test sonucuna göre modeller herhangi bir otokorelasyon içermemektedir. Ayrıca modeller CUSUM ve CUSUMSQ test sonuçlarına göre ele alınan modellerin istikrarlı olduğu tespit edilmiştir. 


\section{SONUÇ}

Ekonomi, okullara göre faiz oranı para talebi üzerinde etkili olan en önemli faktörlerden birisidir. $\mathrm{Bu}$ çalışmada literatürde önemli çalışmalar baz alınarak M2 para arzı, GSYIH, döviz kuru, faiz oranı ve yıllık enflasyon oranını ele alınarak Türkiye için para talep fonksiyonu tahmin edilerek NARDL modeli kullanılmış ve faiz oranının para talebi üzerindeki simetrik etkisi analiz edilmiştir. Bu araştırmada 2005Q4-2019Q3 dönemini kapsayan Türkiye için para talebi fonksiyonu, faiz ile etkileşimini çeyrek veriler kullanılarak belirlenmeye çalışılmıştır. $M_{2}$ para arzı enflasyon oranına bölünerek reel para arzı elde edilmiş, para talebi fonksiyonu NARDL metodu çerçevesinde modellenmiş ve ele alınan değişkinler arasında uzun ve kısa dönemli eşbütünleşik ilişki tespit edilmiştir. Çalışmanın sonucunda elde edilen ampirik bulgulara göre uzun dönemki gelir, faiz ${ }^{\text {pos }}$ oranını doğrusal ve pozitif yönde etkilemişken faiz ${ }^{\text {neg }}$ oranı ve döviz kurunu negatif yönde etkilemiştir. Bu bulgular, etkin para politikası için istikrarlı para talebi fonksiyonu gerektiğini savunan monetarist yaklaşımı desteklemektedir. Türkiye'de para talebinin istikrarlı olduğunu söylenebilir. İstikrarlı bir para talebi, merkez bankasının para politikasını kontrol ederek reel ekonomiyi etkileyebileceğini göstermektedir.

\section{KAYNAKLAR}

[1] Akinkunmi, Mustapha Abiodun. 2004. Money Demand in Developing Countries: A Dynamic Panel Approach.” Ph.D. Fordham University. https://search.proquest.com/pqdtglobal/docview/305187144/ abstract/3BC0039D30AB4819PQ/1 (March 3, 2020).

[2] Akinlo, Anthony. 2006. "The Stability of Money Demand in Nigeria: An Autoregressive Distributed Lag Approach.” Journal of Policy Modeling 28: 445-52.

[3] Al-Badi, Fahad K. A. 2002. "Institutional Changes, Money Demand, and Monetary Policy: The Case of Saudi Arabia." Ph.D. Colorado State University. https://search.proquest.com/pqdtglobal/docview/304797317/ abstract/9B448C9F32DD44BDPQ/1 (March 3, 2020).

[4] Alsahafi, Mamdooh. 2009. "Linear and Non -Linear Techniques for Estimating the Money Demand Function: The Case of Saudi Arabia." Ph.D. University of Kansas. https://search.proquest.com/pqdtglobal/ docview/304916683/abstract/368192FF948840B6PQ/1 (March 3, 2020). 
[5] Bahmani-Oskooee, Mohsen. 1996. "The Black Market Exchange Rate and Demand for Money in Iran." Journal of Macroeconomics 18(1): 17176.

[6] - 2001. "How Stable Is M2 Money Demand Function in Japan?" Japan and the World Economy 13(4): 455-61.

[7] Bahmani-Oskooee, Mohsen, and Hafeez Rehman. 2005. "Stability of the Money Demand Function in Asian Developing Countries." Applied Economics 37(7): 773-92.

[8] Chong, Beng Soon, Ming-Hua Liu, and Keshab Shrestha. 2006. "Monetary Transmission via the Administered Interest Rates Channel." Journal of Banking \& Finance 30(5): 1467-84.

[9] Driscoll, M. J., and J. L. Ford. 1980. "The Stability of the Demand for Money Function and the Predictability of the Effects of Monetary Policy." The Economic Journal 90(360): 867-84.

[10] Dritsakis, Nikolaos. 2012. Structural Breaks, Cointegration and the Demand for Money in Greece. Rochester, NY: Social Science Research Network. SSRN Scholarly Paper. https://papers.ssrn.com/ abstract $=2171561$ (March 3, 2020).

[11] Friedman, Milton. 1966. "Interest Rates and the Demand for Money." The Journal of Law \& Economics 9: 71-85.

[12] Gambacorta, Leonardo. 2008a. "How Do Banks Set Interest Rates?" European Economic Review 52(5): 792-819.

[13] - 2008b. "How Do Banks Set Interest Rates?" European Economic Review 52(5): 792-819.

[14] Greenwood-Nimmo, Matthew, Yongcheol Shin, and Till van Treeck. 2010. The Great Moderation and the Decoupling of Monetary Policy from Long-Term Rates in the U.S. and Germany. IMK at the Hans Boeckler Foundation, Macroeconomic Policy Institute. https://ideas.repec.org/p/ imk/wpaper/15-2010.html (February 18, 2020).

[15] Halicioglu, Ferda \& Ugur, Mehmet, 2005, On stability of the demand for money in a developing OECD country: the case of Turkey. Global Business and Economics Review. 7.203-213.10.1504/GBER.2005.007616. 
[16] Laidler, David E. W. 1993. The Demand for Money: Theories, Evidence, and Problems. HarperCollins College Publishers.

[17] Liao, Weinian. 2005. "Evolution of the Payments System and the Long -Term Demand for Money in Canada." Ph.D. McGill University (Canada). https://search.proquest.com/pqdtglobal/docview/305374232/ abstract/E67AEB88D39347A5PQ/1 (March 3, 2020).

[18] Komijani, A. \& Nazarian, R. (2004). Behavioral pattern of income velocity of money and estimation of its function (The case of Iran). Iranian Economic Review, 9(11), 21-55.

[19] Mishkin, Frederic S. 2004. The Economics of Money, Banking, and Financial Markets 7th Edition (Seventh Edition) by Frederic S. Mishkin. 7 edition. Addison Wesley.

[20] Poole, William. 1970. "Optimal Choice of Monetary Policy Instruments in a Simple Stochastic Macro Model.” The Quarterly Journal of Economics 84(2): 197-216.

[21] Shahrestani, Hamid, and Hosein Sharifi-Renani. 2007. "Demand for Money in Iran: An ARDL Approach.” 83. https://mpra.ub.uni-muenchen. de/11451/ (March 3, 2020).

[22] Shin, Yongcheol, Byungchul Yu, and Matthew Greenwood-Nimmo. 2011. "Modelling Asymmetric Cointegration and Dynamic Multipliers in a Nonlinear ARDL Framework." SSRN Electronic Journal. http://www. ssrn.com/abstract=1807745 (February 10, 2020).

[23] Stern, Liliana V. 2006. "Money Demand, Structural Drift, and Equity Returns." Ph.D. Indiana University. https://search.proquest.com/ pqdtglobal/docview/305335527/abstract/28439AC3F0AD4D56PQ/1 (February 20, 2020).

[24] Tanku, Altin. 2005. "A Study of Two Exchange Rates and One Money Demand." Ph.D. The University of Wisconsin - Milwaukee. https://search.proquest.com/pqdtglobal/docview/305380052/ abstract/3AF844B4EA85437APQ/1 (March 3, 2020). 